\title{
Couniform Modules
}

\author{
Inaam Mohammed Ali*
}

Muna A.Ahmed **

Received 5,September,2011

Accepted 19,February,2012

\begin{abstract}
:
In this paper we introduce and study a new concept named couniform modules, which is a dual notion of uniform modules, where an R-module $\mathrm{M}$ is said to be couniform if every proper submodule $\mathrm{N}$ of $\mathrm{M}$ is either zero or there exists a proper submodule $\mathrm{N}_{1}$ of $\mathrm{N}$ such that $\frac{\mathrm{N}}{\mathrm{N} 1}$ is small submodule of $\frac{\mathrm{M}}{\mathrm{N} 1}$ (denoted by $\frac{\mathrm{N}}{\mathrm{N} 1} \ll \frac{\mathrm{M}}{\mathrm{N} 1}$ )

Also many relationships are given between this class of modules and other related classes of modules. Finally, we consider the hereditary property between R-module M and $\mathrm{R}$-module $\mathrm{R}$ in case $\mathrm{M}$ is couniform.

Keywords: Epiform modules, Hollow modules, Coquasi Dedekind module, Copolyform modules, Small submodules, Semismall submodule, Coessential submodules.
\end{abstract}

\section{Introduction:}

Let $\mathrm{R}$ be a commutative ring with unity and let $\mathrm{M}$ be an R-module. Recall that a submodule $\mathrm{N}$ of $\mathrm{M}$ is said to be small in $\mathrm{M}$, (denoted by $\mathrm{N} \ll \mathrm{M}$ ), if $\mathrm{N}+\mathrm{L} \neq \mathrm{M}$ for every proper submodule $\mathrm{L}$ of $\mathrm{M}$ [1].And $\mathrm{M}$ is called hollow module if every proper submodule of $\mathrm{M}$ is small [2].Hollow module is a dual notion of the uniform module, where an R-module $\mathrm{M}$ is called uniform if every nonzero submodule $\mathrm{N}$ of $\mathrm{M}$ is essential; that is $\mathrm{N} \cap \mathrm{L} \neq 0$ for every nonzero submodule L of M [3]. In this paper another dual notion of uniform module is introduced which we call it couniform module. In section 1 of this paper, we give some basic properties of couniform module. In section 2 , we study the relationships of couniform modules with other types of modules such as hollow modules, epiform modules and coquasi-Dedekind modules. In section 3 , we consider the hereditary property between the ring $\mathrm{R}$ and the R-module $\mathrm{M}$.

\section{CouniformModules - Basic Properties:}

Firstly, we introduce the following concept.

Definition(1.1): A nonzero module $M$ is called couniform, if every proper submodule $\mathrm{N}$ of $\mathrm{M}$ is either zero or there exists a proper submodule $\mathrm{N}_{1}$ of $\mathrm{N}$ such that $\frac{\mathrm{N}}{\mathrm{N} 1} \ll \frac{\mathrm{M}}{\mathrm{N} 1}$

That is for each proper submodule $\mathrm{N}$ of $\mathrm{M}$, either $\mathrm{N}=0$ or there exists a proper submodule $\mathrm{N}_{1}$ of $\mathrm{N}$ such that $\mathrm{N}_{1}$ is coessential submodule of $\mathrm{N}$ in $\mathrm{M}$, where $\mathrm{N}_{1}$ is called coessential submodule of $\mathrm{N}$ in $\mathrm{M}$ if $\frac{\mathrm{N}}{\mathrm{N} 1} \ll \frac{\mathrm{M}}{\mathrm{N} 1} \quad$ [4].

Examples and Remarks (1.2):

1. Every simple module is a couniform module.

2. Semisimple module need not be couniform module, as examples: The $\mathrm{Z}$-module $\mathrm{Z}_{6}, \mathrm{Z}_{10}$ are not couniform modules.

3. Every hollow module is a couniform module. Since for each submodule $\mathrm{N}$ of hollow module $\mathrm{M}, \mathrm{N} \ll \mathrm{M}$. Hence either $\mathrm{N} \neq(0)$ or

* Department of Mathematics, Ibn-Al-Haitham, College of Education, University of Baghdad, Iraq.

** Department of Mathematics. College of Science for Women, University of Baghdad, Iraq 
$\mathrm{N}=(0)$. If $\mathrm{N}=(0)$ then we are done. If $N \neq(0)$ then it is well known that $\frac{\mathrm{N}}{\mathrm{K}} \ll \frac{\mathrm{M}}{\mathrm{K}}$ for all submodule $\mathrm{K}$ of $\mathrm{N}$. Thus $\mathrm{M}$ is a couniform module. And hence every chained module is couniform module. In particular each of the modules $Z_{p^{\infty}}$ and $Z_{8}$ are couniform Z-module.

4. Every proper submodule of a couniform is not coclosed, where $\mathrm{N}_{1}$ is called coclosed submodule of $\mathrm{N}$ in $\mathrm{M}$ if whenever: $\frac{\mathrm{N}}{\mathrm{N} 1} \ll \frac{\mathrm{M}}{\mathrm{N} 1}$ Then $\mathrm{N}=\mathrm{N}_{1}$ [5].

5. The converse of (4) is not true in general, as the following example shows:

The Z- module $\mathrm{Z}$ is not hollow module, but it is a couniform module. To show this, let $\mathrm{N}$ be a nonzero proper submodule of $\mathrm{M}$. If $\mathrm{N}=\langle\mathrm{n}>$, $\mathrm{n} \in \mathrm{Z}_{+}$. By a fundamental theorem of arithmetic, $\mathrm{n}=\mathrm{P}_{1}^{\mathrm{v}_{1}} \mathrm{P}_{2}^{\mathrm{r}_{z}} \mathrm{P}_{3}^{\mathrm{r}_{\mathrm{g}}} \ldots \ldots \ldots \mathrm{P}_{\mathrm{g}}^{\mathrm{r}_{\mathrm{g}}}$, where $\mathrm{P}_{\mathrm{i}}$ is a prime number $\forall \mathrm{i}=$ $1,2, \ldots$. s. Let:

$\mathrm{t}=\max \left\{\mathrm{r}_{1}, \mathrm{r}_{2}, \ldots ., \mathrm{r}_{\mathrm{s}}\right\}+1$. Take $\mathrm{K}=$ $\left(\mathrm{P}_{1}\right)^{\mathrm{t}}\left(\mathrm{P}_{2}\right)^{\mathrm{t}} \ldots \ldots\left(\mathrm{P}_{\mathrm{s}}\right)^{\mathrm{t}}$

Then:

$\frac{\mathrm{N}}{K} \cong<\left(\mathrm{P}_{1}\right)^{\mathrm{t}-\mathrm{r} 1}\left(\mathrm{P}_{2}\right)^{\mathrm{t}-\mathrm{r} 2 \ldots \ldots}\left(\mathrm{P}_{\mathrm{s}}\right)^{\mathrm{t}-\mathrm{r} s}>\ll$ $\mathrm{Z}_{(\mathrm{P} 1) \mathrm{t}(\mathrm{P} 2) \mathrm{t} \ldots . . .(\mathrm{Ps}) \mathrm{t}}$.

Since all submodules of $\mathrm{Z}_{(\mathrm{P} 1) \mathrm{t}}$ (P2)t.....(Ps)t are $<0\rangle, \quad\left\langle\left(\mathrm{P}_{1}\right)\right\rangle$, $\ldots \ldots<\left(\mathrm{P}_{1}^{\mathrm{t}}\right)>,<\left(\mathrm{P}_{2}\right)>\ldots \ldots$, $<\left(\mathrm{P}_{2}^{\mathrm{t}}\right)>, \ldots . .<\left(\mathrm{P}_{\mathrm{s}}\right)>, \quad \ldots . .<\left(\mathrm{P}_{\mathrm{s}}^{\mathrm{t}}\right)>$ $<\left(\mathrm{P}_{1}\right)$

$\left(\mathrm{P}_{2}\right)>\ldots \ldots>,<\left(\mathrm{P}_{1}\right)\left(\mathrm{P}_{2}^{\mathrm{t}}\right), \ldots \ldots \ldots$

But the sum of each one with $\left\langle P_{1}^{t-r_{1}} \ldots \ldots P_{g}^{t-r_{S}}\right\rangle$ is not equal to

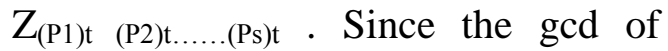
generator of each of one and $\left\langle P_{1}^{t-r_{1}} \ldots \ldots P_{s}^{t-r_{s}}\right\rangle$ is not equal to 1 .

Remark (1.3): The homomorphic image of a couniform module may not be couniform as the following example shows.
The $\mathrm{Z}$-module $\mathrm{Z}$ is couniform module, but if we take the natural epimorphism:

$$
\pi: Z \rightarrow \frac{z}{<6\rangle} \cong Z_{6}
$$

It is clear that $\mathrm{Z}_{6}$ is not couniform $\mathrm{Z}$ module.

Proposition (1.4): A direct summand of a couniform module is couniform.

Proof : Let $\mathrm{N}$ be a direct summand of a couniform module $\mathrm{M}$, thus $\mathrm{M}=\mathrm{N} \oplus \mathrm{K}$ for some submodule $\mathrm{K}$ of $\mathrm{M}$. Let $\mathrm{N}_{1}$ be a nonzero proper submodule of $\mathrm{N}$, then $\mathrm{N}_{1}$ is a proper submodule of $\mathrm{M}$. But $\mathrm{M}$ is a couniform module, so there exists a proper submodule

$\mathrm{N}_{2}$ of $\mathrm{N}_{1}$ such that $\frac{\mathrm{N} 1}{\mathrm{~N} 2} \ll \frac{\mathrm{M}}{\mathrm{N} 2}$, thus $\frac{\mathrm{N} 1}{\mathrm{~N} 2}$ $\ll \frac{\mathrm{N} \oplus \mathrm{K}}{\mathrm{N} 2}$

But $\frac{\mathrm{N} \oplus \mathrm{K}}{\mathrm{N} 2}=\frac{\mathrm{N}}{\mathrm{N} 2} \oplus \frac{\mathrm{N} 2 \oplus \mathrm{K}}{\mathrm{N} 2}$. Thus $\frac{\mathrm{N} 1}{\mathrm{~N} 2} \ll$ $\frac{\mathrm{N}}{\mathrm{N} 2} \oplus \frac{\mathrm{N} 2 \oplus \mathrm{K}}{\mathrm{N} 2} \quad$ i.e $\frac{\mathrm{N} 1}{\mathrm{~N} 2} \oplus(0) \ll \frac{\mathrm{N}}{\mathrm{N} 2} \oplus$ $\frac{\mathrm{N} 2 \oplus \mathrm{K}}{\mathrm{N} 2}$

Hence by [5], $\frac{\mathrm{N} 1}{\mathrm{~N} 2} \ll \frac{\mathrm{N}}{\mathrm{N} 2}$. Therefore $\mathrm{N}$ is a couniform module.

The direct sum of a couniform moe may not be couniform, for example the $\mathrm{Z}$-modules $\mathrm{Z}_{2}, \mathrm{Z}_{3}$, are couniform but $Z_{2} \oplus Z_{3} \cong Z_{6}$ is not couniform.

We claim that a submodule of couniform module need not be couniform, but we cannot find example to ensure that. However, the following proposition deals with the existences of couniform submodules in nonzero Artinian modules, where an R-module $M$ is called Artinian if every descending chain of submodules in $\mathrm{M}$ is stationary [ 1].

Proposition (1.5): Let $\mathrm{M}$ be a nonzero Artinian module, then $\mathrm{M}$ has a submodule which is couniform.

Proof: Let $\mathrm{K}$ be a nonzero submodule of $M$. If $K$ is a couniform then we are done. Otherwise, for each proper submodule $\mathrm{K}_{1}$ of $\mathrm{K}$, the quotient 
module $\frac{\mathrm{K}}{\mathrm{K} 1}$ is not small submodule of $\frac{\mathrm{M}}{\mathrm{K} 1}$.

Now, if $K_{1}$ is a couniform submodule, we are through, otherwise for each proper submodule $\mathrm{K}_{2}$ of $\mathrm{K}_{1}$, the quotient module $\frac{\mathrm{K} 1}{\mathrm{~K} 2}$ is not small submodule of $\frac{\mathrm{M}}{\mathrm{K} 2}$

If we continue in this way we will arrive at a couniform submodule of $\mathrm{M}$ in a finite number of steps, for otherwise there exists an infinite descending chain:

$\mathrm{K} \supset \mathrm{K}_{1} \supset \mathrm{K}_{2} \supset \ldots$

of submodule of $\mathrm{M}$ and this contradicts our assumption.

Recall that an R-module $\mathrm{M}$ is called embedded in $\mathrm{N}$, if there exists a monomorphism f: $\mathrm{M} \rightarrow \mathrm{N}$ [6].

Theorem (1.6): Let $M$ be a couniform module, and let $\mathrm{N}$ be a nonzero small submodule of $M$. If $M$ can be embedded in $\mathrm{N}$, then $\mathrm{N}$ is a couniform.

Proof: Let $\mathrm{N}_{1}$ be a proper submodule of $M$ such that $N_{1} \neq 0$. Since $M$ is a couniform module, so there exists a proper submodule $\mathrm{N}_{2}$ of $\mathrm{N}_{1}$ such that $\frac{\mathrm{N} 1}{\mathrm{~N} 2} \ll \frac{\mathrm{M}}{\mathrm{N} 2}$.

We claim that $\frac{\mathrm{N} 1}{\mathrm{~N} 2} \ll \frac{\mathrm{N}}{\mathrm{N} 2}$. To show this, assume that:

$\frac{\mathrm{N} 1}{\mathrm{~N} 2}+\frac{\mathrm{K}}{\mathrm{N} 2}=\frac{\mathrm{N}}{\mathrm{N} 2}$

For some $\mathrm{N}_{2} \subset \mathrm{K} \subset \mathrm{M}$. Hence $\mathrm{N}_{1}+\mathrm{K}$ $=\mathrm{N}$.

On the other hand, $\mathrm{M}$ can be embedded in $\mathrm{N}$, so there exists a monomorphism f: $M \rightarrow N$. Since $N_{1} \subset N$ and $N \ll M$, so that $\mathrm{N}_{1} \ll \mathrm{M}$. Hence $\mathrm{f}\left(\mathrm{N}_{1}\right) \ll \mathrm{N}$. But $\mathrm{f}\left(\mathrm{N}_{1}\right) \cong \mathrm{N}_{1}$, thus $\mathrm{N}_{1} \ll \mathrm{N}$. It follows that $\mathrm{K}=\mathrm{N}$, and this implies that $\frac{\mathrm{K}}{\mathrm{N} 2}=$ $\frac{\mathrm{N}}{\mathrm{N} 2}$, And so $\frac{\mathrm{N} 1}{\mathrm{~N} 2} \ll \frac{\mathrm{N}}{\mathrm{N} 2}$. Therefore $\mathrm{N}$ is a couniform module.

The Relation Between Couniform Modules and Other Well Known Classes of Modules:
In this section we investigate the relations between couniform modules with other classes of modules such as hollow, epiform, coquasi Dedekind and copolyform modules. In Examples and Remarks (1.2)(5), we see that a couniform module may not be hollow. However we have the following proposition.

Proposition (2.1): Every Artinian couniform module is hollow module.

Proof: Let $M$ be an Artinian couniform module, and let $\mathrm{N}$ be a proper submodule of $\mathrm{M}, \mathrm{N} \neq(0)$. To prove $\mathrm{N}$ is small submodule of $\mathrm{M}$. suppose that $\mathrm{N}$ is not small submodule of $\mathrm{M}$, that is $\mathrm{N}+\mathrm{W}=\mathrm{M}$ for some proper submodule $\mathrm{W}$ of $\mathrm{M}$. Since $\mathrm{M}$ is a couniform module, there exists a proper submodule $\mathrm{N}_{1}$ of $\mathrm{M}$ such that $\frac{\mathrm{N}}{\mathrm{N} 1} \ll \frac{\mathrm{M}}{\mathrm{N} 1}$

Note that $\mathrm{N}_{1} \neq(0)$ because if $\mathrm{N}_{1}=(0)$ then $\mathrm{N}$ is small submodule of $\mathrm{M}$ which is a contradiction. On the other hand, it is easily to show that $\mathrm{N}+\mathrm{W}=\mathrm{M}$, implies that $\mathrm{N}_{1}+\mathrm{W}=\mathrm{M}$, hence $\mathrm{N}_{1}$ is not small submodule of $\mathrm{M}$. Again $\mathrm{N}_{1}$ is a non zero proper submodule of $\mathrm{M}$, so there exists a proper submodule $\mathrm{N}_{2}$ of $\mathrm{N}_{1}$ such that $\frac{\mathrm{N} 1}{\mathrm{~N} 2} \ll \frac{\mathrm{M}}{\mathrm{N} 2}$. Hence $\mathrm{N}_{2} \neq(0)$. Since $\mathrm{N}_{1}+\mathrm{W}=\mathrm{M}$, then $\mathrm{N}_{2}+\mathrm{W}=\mathrm{M}$. Repeating this process until we have infinite strictly descending chain:

$$
\mathrm{N} \supset \mathrm{N}_{1} \supset \mathrm{N}_{2} \supset
$$

of submodules in $\mathrm{N}$. But this is a contradiction since $\mathrm{N}$ is Artinian [1], thus our assumption is false, therefore $\mathrm{N} \ll \mathrm{M}$, that is $\mathrm{M}$ is a hollow module.

Remark (2.2): The condition "M is Artinian" cannot be dropped from the previous proposition, since $\mathrm{Z}$ is a couniform as Z-module which is not Artinian and not hollow module. Note that each of the $\mathrm{Z}$-modules $\mathrm{Z}_{6}$ and $\mathrm{Z}_{10}$ is not couniform module (see Examples and Remarks 1.2(3)), and they are Artinian Z-modules.

Definition (2.3)[7]: A proper submodule $\mathrm{N}$ of an $\mathrm{R}$-module $\mathrm{M}$ is 
called semismall, if either $\mathrm{N}=0$ or for each nonzero submodule $\mathrm{K}$ of $\mathrm{N}$ :

$$
\frac{\mathrm{N}}{K} \ll \frac{\mathrm{M}}{\mathrm{K}}
$$

And an R-module $\mathrm{M}$ is called semihollow, if every proper submodule of $\mathrm{M}$ is semismall. It is clear that every hollow module is semihollow, but the converse is not true, see [7]. So we have the following.

Remark (2.4): A semihollow module need not to be couniform module, for example $Z_{6}$ as $\mathrm{Z}$-module is semihollow module but it is not couniform

Remark (2.5): A couniform module need not to be semihollow, for example the $\mathrm{Z}$ as $\mathrm{Z}$ module is a couniform, but not semihollow module. However, if $\mathrm{M}$ satisfies d.c.c on non small submodules, then a couniform module can be semihollow as the following proposition shows.

Proposition (2.6): Let $\mathrm{M}$ be a module which satisfies d.c.c on non small submodules. If $\mathrm{M}$ is a couniform module then $\mathrm{M}$ is semihollow.

Proof: Let $\mathrm{N}$ be a zero proper submodule of $M$. Suppose $M$ is not semihollow, there exist a proper submodule $\mathrm{N}_{1}$ of $\mathrm{N}$ such that $\frac{\mathrm{N}}{\mathrm{N} 1}$ is not small submodule of $\frac{\mathrm{M}}{\mathrm{N} 1}$. Hence $\mathrm{N}$ is not small submodule of $M$, so there exists a proper submodule $B$ of $M$ such that $\mathrm{N}+\mathrm{B}=\mathrm{M}$. But $\mathrm{M}$ is couniform, so there exists a proper submodule $\mathrm{N}_{2}$ of $\mathrm{N}$ such that $\frac{\mathrm{N}}{\mathrm{N} 2} \ll \frac{\mathrm{M}}{\mathrm{N} 2}$.

It is clear that $\mathrm{N}_{2} \neq 0$, because if $\mathrm{N}_{2}=0$ then $\mathrm{N} \ll \mathrm{M}$. On the other hand $\mathrm{N}+\mathrm{B}=$ $M$ implies that $N_{2}+B=M$, that is $N_{2}$ is not small submodule of $\mathrm{M}$. Again, $\mathrm{N}_{2} \neq$ 0 implies that there exists a proper submodule $\mathrm{N}_{3}$ of $\mathrm{N}_{2}$ such that $\frac{\mathrm{N} 2}{\mathrm{~N} 3} \ll$ $\frac{\mathrm{M}}{\mathrm{N} 3}$. It follows that $\mathrm{N}_{3} \neq 0$.

But $\mathrm{N}_{2}+\mathrm{B}=\mathrm{M}$ implies that $\mathrm{N}_{3}+\mathrm{B}$ $=\mathrm{M}$. Repeating this process until we get strictly descending chain of non small submodule in N. But this contradicts our assumption. Thus $\mathrm{N}$ is semismall submodule of $\mathrm{M}$. That is $\mathrm{M}$ is a semihollow module. Recall that an $\mathrm{R}$-module $\mathrm{M}$ is called noncosingular module, if for any nonzero module $\mathrm{N}$ and for every nonzero homomorphism $\mathrm{f}: \mathrm{M} \longrightarrow \mathrm{N}, \operatorname{Im} \mathrm{f}$ is not small submodule of N [8]. And an R-module $M$ is called epiform, if every nonzero homomorphism $\mathrm{f}: \mathrm{M} \longrightarrow \frac{\mathrm{M}}{\mathrm{K}}$ with $\mathrm{K}$ a proper submodule of $\mathrm{M}$ is an epimorphism [9]. It was proved in [9], that if an R-module $\mathrm{M}$ is noncosingular, then $\mathrm{M}$ is an epiform if and only if $\mathrm{M}$ is hollow module, so we have the following.

Proposition (2.7): Let $M$ be an Artinian and noncosingular module. If $M$ is a couniform module, then $M$ is epiform module.

Proof: Since $M$ is an Artinian and couniform module then by prop.(2.1), $M$ is hollow module. But $M$ is noncosingular, thus it is an epiform module [9, Prop. (2.5)]. Recall that an $\mathrm{R}$-module $\mathrm{M}$ is called cosemisimple, if $\operatorname{Rad}\left(\frac{\mathrm{M}}{\mathrm{K}}\right)=0$, for all submodules $\mathrm{K}$ of M [10].

Corollary (2.8): Let $\mathrm{M}$ be an Artinian and cosemisimple module. If $\mathrm{M}$ is a couniform module, then $\mathrm{M}$ is epiform module.

Proof: By using Prop. (2.1) and [9, Prop. (2.6)].

Recall that a nonzero module $\mathrm{M}$ is called coquasi Dedekind, if every proper submodule of $\mathrm{M}$ is coquasi invertible, where a proper submodule $\mathrm{N}$ of $\mathrm{M}$ is called coquasi invertible, if $\operatorname{Hom}_{\mathrm{R}}(\mathrm{M}, \mathrm{N})=0$ [11].

Theorem (2.9): Let $M$ be an Rmodule. If $\mathrm{M}$ is an epiform module then $\mathrm{M}$ is couniform and coquasi Dedekind module.

Proof: Since every epiform module is hollow [9], then by (1.2)(4), $\mathrm{M}$ is a couniform module. On the other hand by $[9, \quad \operatorname{Rem} .(1.2)]$, each nonzero 
homomorphism in $\operatorname{End}_{\mathrm{R}}(\mathrm{M})$ is epimorphism. By [11, Th.(2.1.4) ], M is coquasi Dedekind.The following example shows that a coquasi Dedekind may not be epiform module, before that let us recall that a submodule $\mathrm{N}$ of an $\mathrm{R}$ - module $\mathrm{M}$ is called corational submodule in $\mathrm{M}$ if $\operatorname{Hom}_{R}\left(\mathrm{M}, \frac{\mathrm{N}}{\mathrm{K}}\right)=0$ for all submodule $\mathrm{K}$ of $\mathrm{M}$ such that $\mathrm{K} \subseteq \mathrm{N}$ [11].

Example (2.10): The Z-module $\mathrm{Q}$ is a coquasi Dedekind but not epiform module, since if we assume that then by [9], every proper submodule of $\mathrm{Q}$ is corational, hence it is small submodule[11], which implies that Q is a hollow module[2]. But this is a contradiction.However, under the class of self projective modules we have the following, but first recall that an Rmodule $M$ is called self projective, if $\mathrm{M}$ is $\mathrm{M}$ - projective [3].

proposition (2.11): Let $M$ be a self projective R-module. Then $\mathrm{M}$ is an epiform module if and only if $M$ is coquasi Dedekind module.

Proof: $\mathrm{M}$ is an epiform module $\leftrightarrow$ each proper submodule of $\mathrm{M}$ is a corational [9] $\leftrightarrow$ each proper submodule of $\mathrm{M}$ is coquasi invertible [11, Th.(1.2.13)] $\leftrightarrow \mathrm{M}$ is a coquasi Dedekind module [11].

Corollary (2.12): Let $M$ be a self projective R-module, then the following statement are equivalent:

1. $\mathrm{M}$ is an epiform module.

2. $\mathrm{M}$ is a coquasi Dedekind module.

3. $\mathrm{M}$ is a couniform and coquasi Dedekind module.

Proof: $\quad(1) \Rightarrow$ (3): By Th. (2.9).

(3) $\Rightarrow(2)$ : It is clear.

$(2) \Rightarrow(1)$ : It follows from

Prop. (2.11).

Note that the Z-module Q is not self projective [12], so it is natural to see that $\mathrm{Q}$ is a coquasi Dedekind and not epiform module. On the other hand, the Z-module $\mathrm{Z}_{4}$ is a self projective and not coquasi Dedekind module, thus $\mathrm{Z}_{4}$ must be not epiform.

Now under the class of multiplication module the two concepts of epiform and coquasi Dedekind module are equivalent, where an R-module $\mathrm{M}$ is called multiplication, if each submodule $\mathrm{N}$ of $\mathrm{M}$ can be written as the form $\mathrm{N}=\mathrm{IM}$, for some ideal Iof R [13].

proposition (2.13): Let $M$ be a multiplication R-module. Then $\mathrm{M}$ is an epiform module if and only if $M$ is a coquasi Dedekind module.

Proof: $\Rightarrow$ ) By Th.(2.9)

$\Leftarrow) \mathrm{M}$ is a coquasi Dedekind module implies that every proper submodule of $\mathrm{M}$ is acoquasi invertible [11, Th.(1.2.13)]. But $\mathrm{M}$ is multiplication module, so by $[11$, Th.(1.2.7)], $\mathrm{N}$ is a corational. Thus $\mathrm{M}$ is an epiform module [9].

Corollary (2.14): Let $M$ be a multiplication R-module. The following statements are equivalent:

1. $\mathrm{M}$ is an epiform module.

2. $\mathbf{M}$ is a coquasi Dedekind module.

3. $\mathrm{M}$ is a couniform and coquasi Dedekind module.

Corollary (2.15): Let $M$ be self projective module and $\mathrm{J}(\operatorname{End}(\mathrm{M})=0$, then:

1. $\mathrm{M}$ is an epiform module.

2. $\mathrm{M}$ is a hollow module.

3. $\mathrm{M}$ is a coquasi Dedekind module.

4. $\mathrm{M}$ is a couniform and coquasi Dedekind module.

Proof: $\quad(1) \Leftrightarrow(3) \Leftrightarrow(4)$ : Follows from Cor. (2.14)

Th.(1.2.16)].

$$
(2) \Leftrightarrow \quad(3): \quad \text { By } \quad[11 \text {, }
$$

Recall that an R-module $\mathrm{M}$ is called copolyform, if $\operatorname{Hom}_{R}\left(M, \frac{\mathrm{N}}{\mathrm{K}}\right)=0$, for all submodule $\mathrm{N}$ of $\mathrm{M}$ with $\mathrm{K}$ $\subseteq \mathrm{N} \ll \mathrm{M}$ [14]. The following proposition appeared in [9]. 
Proposition (2.16) [9]: An R-module $M$ is epiform if and only if $M$ is a hollow and copolyform module.

Corollary (2.17): Let $M$ be an Rmodule. If $\mathrm{M}$ is epiform then $\mathrm{M}$ is couniform and copolyform module.

Proof: Since $\mathrm{M}$ is epiform module, then by Th.(2.9), $\mathrm{M}$ is a couniform module. Also by [9], $\mathrm{M}$ is copolyform module.

The converse of Cor.(2.17) is not true in general, for example $\mathrm{Z}$ as $\mathrm{Z}$ module is a couniform and copolyform module but not epiform module.

\section{Hereditary of couniform modules}

This section deals with the hereditary of a couniform property. That is the transitivity of a couniform property between $M$ and the ring $R$ which defined on it. Note that a ring $R$ is called couniform ring if $R$ is couniform R-module. Let us begin by the following theorem.

Theorem (3.1): Let $\mathrm{R}$ be a ring, and let $M$ be a finitely generated faithful multiplication R-module. Then $\mathrm{M}$ is couniform module if and only if $\mathrm{R}$ is a couniform module.

Proof: $\Rightarrow$ ) Let I be a nonzero proper ideal of R. Put $I M=N$. Then $N$ is a nonzero proper submodule of $\mathrm{M}$. Since $\mathrm{M}$ is a couniform module, so $\exists \mathrm{N}_{1}<\mathrm{N}$ such that $\frac{\mathrm{N}}{\mathrm{N} 1} \ll \frac{\mathrm{M}}{\mathrm{N} 1}$.

Since $M$ is a multiplication module, then $\mathrm{N}_{1}=\mathrm{JM}$ for some proper ideal $\mathrm{J}$ of $\mathrm{R}$, therefore:

$\frac{\mathbb{I M}}{\mathbb{I M}} \ll \frac{\mathrm{M}}{\mathbb{I M}}$. We claim that $\frac{\mathbb{I}}{\mathbb{I}} \ll \frac{\mathrm{R}}{\mathbb{I}}$. To show this assume that $\frac{\mathbb{I}}{\mathrm{I}}+\frac{\mathrm{K}}{\mathrm{I}}=\frac{\mathrm{R}}{\mathrm{I}}$ for some $\frac{\mathbb{K}}{\mathrm{J}}<\frac{\mathbb{R}}{\mathbb{I}}$.

Hence $\mathrm{I}+\mathrm{K}=\mathrm{R}$ and this implies that $(\mathrm{I}+\mathrm{K}) \mathrm{M}=\mathrm{M}$, so $\mathrm{IM}+\mathrm{KM}=\mathrm{M}$. It follows that:

$\frac{\mathrm{IM}+\mathrm{KM}}{\mathrm{IM}} \ll \frac{\mathrm{M}}{\mathrm{JM}}$. Hence $\frac{\mathrm{IM}}{\sqrt{\mathrm{M}}}+\frac{\mathrm{KM}}{\sqrt{\mathrm{M}}}=\frac{\mathrm{M}}{\mathrm{JM}}$. But $\frac{\mathrm{IM}}{\mathrm{JM}} \ll \frac{\mathrm{M}}{\mathrm{JM}}$. Hence $\frac{\mathrm{KM}}{\mathrm{JM}}=\frac{\mathrm{M}}{\mathrm{JM}}$. Thus KM $=\mathrm{M}=\mathrm{RM}$.
Since $\mathrm{M}$ is a faithful finitely generated multiplication module, therefore $\mathrm{K}=$ $R$, and hence $\frac{\mathbb{I}}{\mathbb{I}} \ll \frac{\mathbb{R}}{\mathbb{I}}$. That is $R$ is a couniform module.

$\Leftarrow)$ Let $\mathrm{N}$ be a nonzero proper submodule of $M$. Since $M$ is a multiplication module, so $\mathrm{N}=\mathrm{IM}$ for some ideal $I$ of $R$ [13]. But $M$ is a finitely generated multiplication module, so $\mathrm{I}$ is a nonzero proper ideal of $\mathrm{R}$ [13]. On the other hand, $\mathrm{R}$ is a couniform R-module, therefore there exists a proper ideal $\mathrm{J}$ of $\mathrm{I}$ such that I $\ll \frac{\mathrm{R}}{\mathrm{I}}$. Also $\mathrm{J}<\mathrm{I}$ implies that $\mathrm{JM}<$ IM. We claim that $\frac{\mathrm{IM}}{\mathrm{TM}} \ll \frac{\mathrm{RM}}{\mathrm{IM}}$. To show this assume that $\frac{\mathbb{M M}}{\mathbb{N M}}+\frac{\mathrm{W}}{\mathrm{IM}}=\frac{\mathrm{M}}{\mathrm{IM}}$ for some $\frac{\mathrm{w}}{\mathrm{NM}}<\frac{\mathrm{M}}{\mathrm{JM}}$, hence $\mathrm{IM}+\mathrm{W}=\mathrm{M}$.

But $\mathrm{W}=\mathrm{KM}$ for some ideal $\mathrm{K}$ of $\mathrm{R}$, so $\mathrm{IM}+\mathrm{KM}=\mathrm{M}$ which implies that $(\mathrm{I}+$ K) $\mathrm{M}=\mathrm{M}$. BY [13], I + K = R. Hence $: \frac{\mathbb{I}+\mathrm{K}}{\mathrm{I}}=\frac{\mathrm{R}}{\mathrm{J}}$ That is $\frac{\mathbb{I}}{\mathrm{I}}+\frac{\mathrm{K}}{\mathrm{I}}=\frac{\mathrm{R}}{\mathrm{J}}$. But $\frac{\mathbb{I}}{\mathrm{I}} \ll \frac{\mathrm{R}}{\mathrm{I}}$ Thus $\frac{\mathrm{K}}{\mathrm{I}}=\frac{\mathrm{R}}{\mathrm{I}}$. Hence $\mathrm{K}=\mathrm{R}$,

and so $\mathrm{W}=\mathrm{KM}=\mathrm{M}$. Thus $\frac{\mathrm{W}}{\mathrm{JM}}=\frac{\mathrm{M}}{\mathrm{JM}}$ and $\frac{\mathrm{IM}}{\mathbb{M}} \ll \frac{\mathrm{M}}{\mathbb{N}}$. Therefore $\mathrm{M}$ is a couniform module.

From the above theorem we conclude the following result, before that we need the following lemma.

Lemma (3.2): let $M$ be a finitely generated faithful multiplication $\mathrm{R}$ module. Then $\mathrm{M}$ is coquasi Dedekind module if and only if $R$ is a coquasi Dedekind R-module.

Proof: $\Rightarrow$ Let $f \in \operatorname{End}(R), f \neq 0$. Then $f(r)=r a$ for some $a \in R, a \neq 0$.

Define $\mathrm{g}: \mathrm{M} \rightarrow \mathrm{M}$ bY $\mathrm{g}(\mathrm{m})=\mathrm{am}$. Then $\mathrm{g} \neq 0$, hence $\mathrm{g}$ is an epimorphism that is $\mathrm{aM}=\mathrm{M}$, which implies that $\langle\mathrm{a}\rangle=$ $\mathrm{R}$, i.e $\mathrm{f}$ is an epimorphism.

$\Leftarrow)$ Let $\mathrm{f} \in \operatorname{End}(\mathrm{M}), \mathrm{f} \neq 0$. Since $\mathrm{M}$ is a finitely generated multiplication 
module, so for each $\mathrm{m} \in \mathrm{M}$, there exist a nonzero $a \in R$ s.t $\mathrm{f}(\mathrm{m})=\mathrm{am}$.

Define $g: R \rightarrow R$ by $g(r)=r a . ~ g \neq 0$, hence $\mathrm{g}$ is an epimorphism; that is $\langle\mathrm{a}\rangle=\mathrm{g}(\mathrm{R})=\mathrm{R}$ and so $\mathrm{f}(\mathrm{M})=$ $<\mathrm{a}>\mathrm{M}=\mathrm{RM}=\mathrm{M}$. Thus $\mathrm{f}$ is an epimorphism that is $M$ is a coquasi Dedekind module.

Corollary (3.3): let $\mathrm{M}$ be a finitely generated faithful multiplication Rmodule. Then the following statements are equivalent.

1. $\mathrm{M}$ is an epiform module.

2. $\mathrm{M}$ is a coquasi Dedekind module.

3. $\mathrm{R}$ is an epiform ring.

4. $\mathrm{R}$ is a coquasi Dedekind ring.

5. $\mathrm{M}$ is a couniform and coquasi Dedekind module.

6. $\mathrm{R}$ is a couniform and coquasi Dedekind ring.

7. $\operatorname{End}_{R}(M)$ is a couniform and coquasi Dedekind ring.

Proof: $(1) \Leftrightarrow(2) \Leftrightarrow(5)$ : It follows from Cor.(2.14).

$(3) \Leftrightarrow(4) \Leftrightarrow(6)$ : It follows from Cor.(2.14), since $\mathrm{R}$ is multiplication module.

(5) $\Leftrightarrow$ (6): It follows from

Th.(3.1) and Lemma(3.2).

$(6) \Leftrightarrow(7)$ : Since $M$ is a faithful multiplication $\mathrm{R}$ module, then $\operatorname{End}_{R}(M)=R$ and hence the result obtained.

\section{References:}

1. Kasch, F. 1982, Modules and rings, Academic Press Inc. London.

2. Fleury, P.P. 1974, Hollow modules and local endomorphism rings, Pacific J. Math., 53, 379-385.3. Dung, N.V., Huynh, D.V., Smith, P.F. and Wisbauer R..1994, Extending modules,
Pitman Research Note in Math., Series 313 Longman Harlow.

4. Courter R.C.1966, The maximal corational extension by a module, Can.J.Math.,18, 453-962.

5. Golan, J.S. 1971, Quasi-semiperfect modules, Quart.J.Math.,Oxford, 22, 173-182.

6. Hungerford, T.W. 1974, Algebra, Springer-Verlag, Berlin.

7. Ali, I.M. and Mahmood, L.S. 2009, Semismall submodules and semi-lfting modules, Proceeding of $3^{\text {rd }}$ scientific conference of the college of science, Univ. of Baghdad, 24-26 March.

8.Ganesan, L. and Vanaja, N. 2002, Modules for which every submodule has a unique coclosure, Comm. Algebra, 30(5), 2355-2377.

9. Al-Hashimi, B.A. and Ahmed M.A. 2010, Some results on epiform modules, J. Al-Anbar, Univ. for pure sci., 4(3), 54-56.

10. Anderson, F.W. and Fuller, K.R. 1992, Rings and categories of modules, Springer-Verlag, New York.

11. Yasseen, S.M. 2003, CoquasiDedekind modules, Ph.D. Thesis, College of Science, Univ. of Baghdad.

12. Abbas, A. 2006, Some results on $\pi$ projective modules, Ph.D.Thesis, College of Science, Univ. of Baghdad.

13. El-Bast, Z. A. and Smith, P. F. 1988, Multiplication modules, Comm. In Algebra, 16, 755-779.

14. Lomp, C. 1996, On dual Goldie dimension, Diplomartbeit M.Sc. Thesis, University of Düsseldorf, Germany. 


\section{المقاسات المنتظمة المضادة}

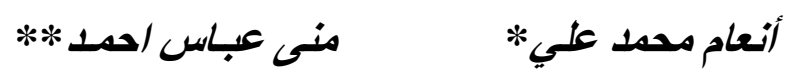

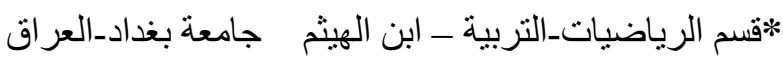

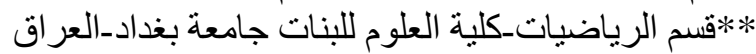

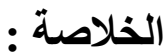

في هذا البحث قدمنا ودرسنا مفهوم جديد اطلقنا عليه اسم الدقاس المنتظم المضاد الذي هو رديف للمقاس المنتظم،

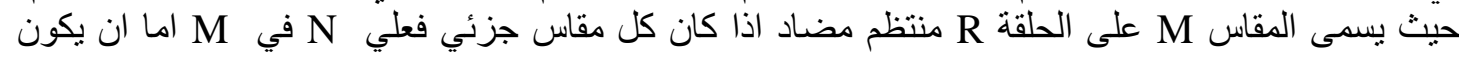

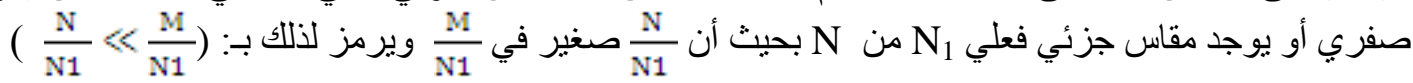

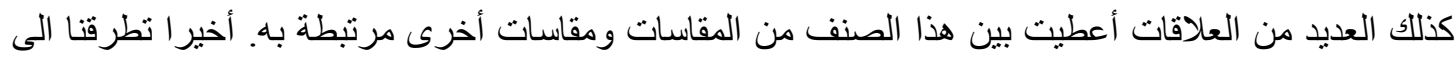

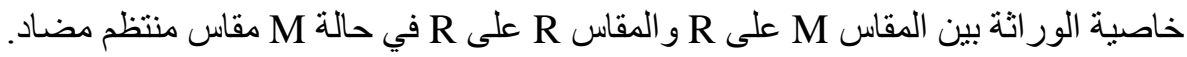

